\title{
Acoustic Neuromas: Management of 204 Cases
}

\author{
Charles H. Tator
}

\begin{abstract}
This paper reviews the author's personal experience with the management of 204 patients with the clinical diagnosis of acoustic neuroma. Craniotomy was performed in 181 of these cases, all of whom were proven to have acoustic neuromas. The remaining 23 cases with the clinical diagnosis of acoustic neuroma did not have tumour surgery, mainly because of advanced age or concurrent disease, although some required shunts for hydrocephalus. The management of acoustic neuromas including the selection of surgical approach depends upon the patient's age and general health, the size and growth direction of the tumour, previous surgical attempts at removal, and the presence of hydrocephalus, a contralateral tumour, and serviceable hearing. Twenty-nine other cases with the clinical diagnosis of acoustic neuroma underwent craniotomy: 20 had petrous or tentorial meningiomas encroaching on the porus acousticus or growing in the internal auditory canal, six had neuromas of the seventh cranial nerve and three had arachnoiditis occluding the internal auditory canal.
\end{abstract}

RÉSUMÉ: Neurinomes acoustiques: traitement de 204 cas L'auteur revoit son expérience personnelle dans le traitement de 204 cas de neurinomes acoustiques. Une craniotomie fut faite chez 181 de ces cas, tous ayant un neurinome acoustique. Les derniers 23 cas, dont le diagnostic clinique était de neurinome acoustique, n'ont pas été opérés pour cette tumeur, surtout à cause de leur âge avancé ou de maladies simultanées, mais certains ont dû recevoir une dérivation pour l'hydrocéphalie. La conduite à suivre devant un cas de neurinome acoustique, incluant la sélection de l'approche chirurgicale, dépend de l'âge et de l'état de santé du patient, de la grosseur et de la direction de la croissance de la tumeur, des tentatives chirurgicales antérieures, de la présence d'hydrocéphalie, d'une tumeur contralatérale et d'une ouie compétente. Vingt-neuf autres cas avec le diagnostic clinique de neurinome acoustique ont aussi été opérés: 20 cas avaient des méningiomes pétreux ou tentoriels qui envahissaient le trou acoustique ou le canal auditif interne; 6 cas avaient un neurinome du septième nerf cranien et chez trois cas une arachnoïdite fermait le canal auditif interne.

Can. J. Neurol. Sci. 1985: 12:353-357

The management of patients with acoustic neuromas has changed markedly over the past several decades due largely to several technological advances which allow earlier diagnosis when the tumours are smaller, and better surgical results due to a reduction in operative trauma. There have been two previous large series of acoustic neuromas reported from Canadian centres. In 1955, McKenzie and Alexander ${ }^{1}$ published a series of 142 cases, and in 1967, Drake ${ }^{2}$ published a series of 30 cases. In the first series, the remarkable accomplishment was the reduction in surgical mortality and major morbidity, especially since most of the tumours were of large size. The mortality rate was reduced to $12.1 \%$ in 66 consecutive cases with complete removal. In the second series, the surgical results were distinguished by the larger percentage of complete removals and the preservation of the surrounding structures, especially the facial nerve, due largely to the use of microsurgical techniques. In both of these series of cases the classical suboccipital approach was used exclusively, and indeed, in many centres this is still the only approach used. Since the late 1970's, the author has been involved in a team approach with a neuro-otologist for the treatment of acoustic neuromas, initially with J.M. Nedzelski at Sunnybrook Medical Centre and currently with D.A. Charles at Toronto Western Hospital. It is my belief that this team approach has allowed further improvements in surgical results, mainly because of better access to the tumours through a variety of temporal and suboccipital approaches.

This paper describes the author's personal experience from 1970-85 with the management of 204 consecutive patients with the clinical diagnosis of acoustic neuroma, 181 of whom underwent craniotomy for tumour removal, and acoustic neuroma was confirmed in all. Twenty-three patients have not had tumour surgery, generally due to advanced age or concurrent disease, but some have had shunts for hydrocephalus. Some of the acoustic neuroma patients have been described in previous reports ${ }^{3,4}$. In addition, 29 other operative cases are described in whom the clinical diagnosis was acoustic neuroma, but in whom other pathological lesions were found.

\section{Methods and CaSe Material}

\section{Patient Selection}

Only those cases personally managed by the author are included in this study. Most patients were treated at Sunnybrook Medical Centre and the remainder at the Toronto Western Hospital. For inclusion in the study the preoperative or operative diagnosis must have been acoustic neuroma. In all, 233 patients treated 
Table 1: Acoustic Neuromas: Criteria for Selection of Management

1. Age of patient

2. Size of tumour

3. Medical factors - other diseases - obesity

4. Previous surgery

5. Presence of hydrocephalus

6. Presence of serviceable hearing

7. Presence of bilateral tumours

8. Anterior or posterior tumour location

Table 2: Acoustic Neuromas: Classification of Patients into Management Groups

1. Elderly patients - over 70 years

2. Patients with smaller tumours and serviceable hearing

3. Patients with smaller tumours and poor hearing

4. Patients with larger tumours, regardless of hearing

Definitions:

1. Serviceable hearing - Speech reception threshold less than 50 decibels, and

- speech discrimination score more than $60 \%$

2. "Smaller" tumours $-2.5 \mathrm{~cm}$ or less

3. "Larger" tumours -3.0 . $\mathrm{cm}$ or more

between 1970 and 1985 were analysed and this included the 181 consecutive acoustic neuromas surgically treated by the author.

\section{Selection of Management}

Tables 1-3 outline the criteria used for selection of either operative or nonoperative management and the criteria for selection of the surgical approach. Elderly patients with acoustic neuromas were managed in at least three different ways according to their symptoms and clinical status. Firstly, those with small "asymptomatic" tumours were treated expectantly. Usually, these patients had only unilateral hearing loss, tinnitus or mild dizziness, and they were followed by yearly clinical and CT examinations. The second group of elderly patients had large tumours and symptomatic hydrocephalus, and were treated with CSF shunts and followed closely. The third group included those with large tumours, and progressive symptoms without hydrocephalus, and they were treated surgically generally with complete excision, although partial excision was used as a planned procedure in those with added surgical risk factors, or as an "unplanned" procedure if the tumour was adherent to the brainstem or adjacent cranial nerves.

If the patient had had previous surgery and a second operation was required, a "fresh" operative approach was generally selected. In patients with hydrocephalus, a temporary ventricular drain was inserted at the time of tumour surgery rather than a preoperative permanent shunt which proved unnecessary in most.

The standard suboccipital approach as described previously ${ }^{4.5}$ was selected for patients with small tumours $(2.5 \mathrm{~cm}$ or less) and serviceable hearing (speech reception threshold less than
Table 3: Acoustic Neuromas: The Currently Used Operative Approaches and the Usual Indications

1. Suboccipital approach - smaller tumours with serviceable hearing.

2. Translabyrinthine approach - smaller tumours with poor hearing.

3. Expanded translabyrinthine approach - larger tumours, regardless of hearing.

Table 4: Operative Approach — 210 Cases

\begin{tabular}{|c|c|c|c|c|}
\hline Diagnosis & $\begin{array}{c}\text { Sub- } \\
\text { occipital }\end{array}$ & $\begin{array}{c}\text { Trans- } \\
\text { labyrinthine }\end{array}$ & $\begin{array}{c}\text { Expanded } \\
\text { Trans- } \\
\text { labyrinthine }\end{array}$ & Total \\
\hline \multicolumn{5}{|l|}{ Acoustic } \\
\hline Neuroma & 82 & 33 & 66 & 181 \\
\hline Meningioma & 12 & 1 & 7 & 20 \\
\hline \multicolumn{5}{|l|}{ Facial } \\
\hline Neuroma & 1 & 1 & 4 & 6 \\
\hline \multirow[t]{2}{*}{ Arachnoiditis } & 2 & 1 & 0 & 3 \\
\hline & 97 & 36 & 77 & 210 \\
\hline
\end{tabular}

50 decibels, and speech discrimination score more than $60 \%$ ), while the translabyrinthine approach ${ }^{6}$ was selected for patients with smaller tumours and poor hearing (Table 2). For patients with larger tumours $(3.0 \mathrm{~cm}$ or more) regardless of hearing ability, the expanded translabyrinthine approach as described previously ${ }^{3}$ was generally used (Table 3). For those with bilateral tumours or other special circumstances including contralateral hearing impairment, previous surgery, or unusual growth direction of the tumour, the approach was individualized. For example, one patient had a suboccipital approach to remove a very large posterior and inferior extension of the tumour followed in two weeks by an expanded translabyrinthine approach for a very large anterior and superior extension of the tumour through the tentorial hiatus into the middle fossa. Table 3 shows the three commonly used approaches and the usual indications.

\section{RESULTS}

Table 4 shows that of the 210 patients operated upon, 181 were proven to have acoustic neuromas. Twenty additional patients were found to have meningiomas and all these arose from either the posterior aspect of the petrous temporal bone or the adjacent tentorial margin or both, and almost all extended through the porus acousticus for a variable distance into the internal auditory canal. Six patients had neuromas of the seventh cranial nerve, and three had arachnoidal scarring which obliterated the porus and internal auditory canal. In the 210 operative cases, the suboccipital, translabyrinthine and expanded translabyrinthine approaches were used in 97,36 and 77 cases, respectively. 
The acoustic neuromas were completely excised in 169 patients. A planned partial excision was performed in seven patients most of whom were elderly patients with very large tumours. In the remaining five, a planned complete excision was discontinued because of intra-operative difficulties, and most of these were early in the series. In the 181 acoustic neuroma patients there were two operative deaths (1.1\%). The first was in 1976 and followed suboccipital excision of a $6.0 \mathrm{~cm}$ tumour. The patient awoke intact promptly following surgery but then rapidly deteriorated due to a subdural haematoma. In spite of evacuation of the clot, the patient died five days later from the effects of brainstem compression. The second death occurred in 1978 in a patient who had had three previous partial excisions of an acoustic neuroma in other hospitals. After total excision of his $5.0 \mathrm{~cm}$ tumour through an expanded translabyrinthine approach, he was recovering satisfactorily, and then developed a CSF leak and meningitis. After treatment of the meningitis and operative closure of the leak, the patient again recovered well and was ambulatory. Then, several days later while in the hospital, he fell to the floor, possibly due to a seizure, and sustained a major brainstem injury from which he died six weeks later. There were no operative deaths in the other 29 patients listed in Table 4.

Preservation of seventh and eighth nerve function in the 181 acoustic neuroma patients will be the subject of a separate report. In general, preservation was directly related to tumour size and the severity of preoperative impairment. With smaller tumours, preservation of the continuity of the seventh nerve was achieved in almost all cases, while with larger tumours anatomical continuity was still possible in most, and functional recovery was present in approximately two-thirds as described previously ${ }^{3}$. That report described the first 14 acoustic neuromas $2.5 \mathrm{~cm}$ or larger treated by the expanded translabyrinthine

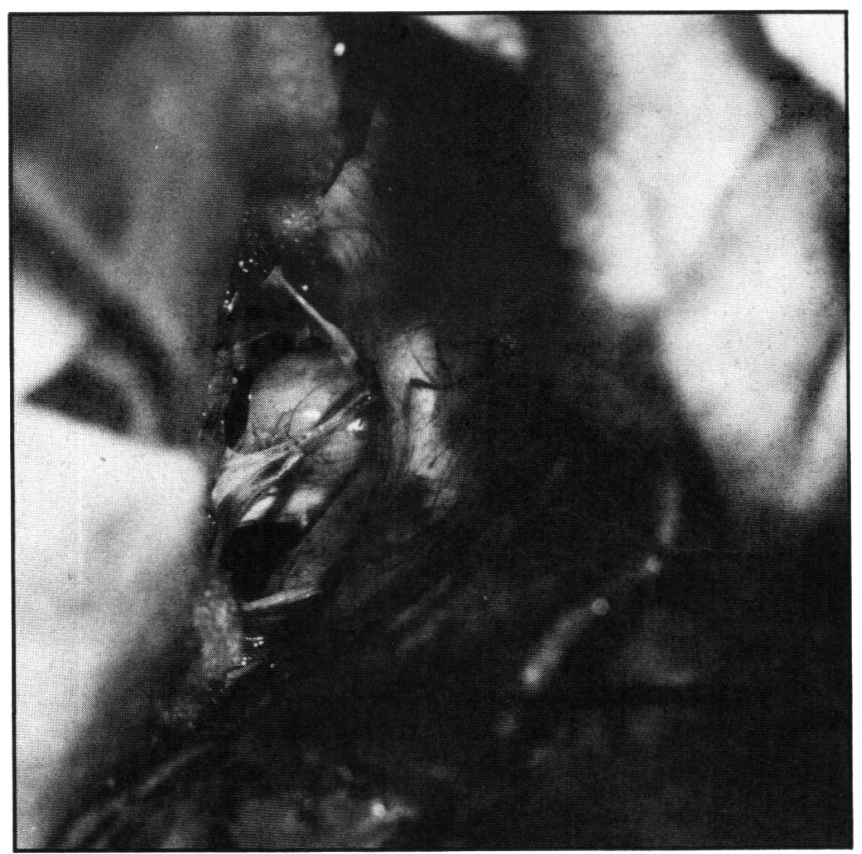

Figure 1 - A small right acoustic neuroma is seen in the CPA adjacent to the porus. The arachnoid over the tumour was divided and the cerebellum retracted medially (the retractor is at the upper left). The cochleo-vestibular bundle is seen along the inferior aspect of the tumour. approach (included in the 181 cases). The facial nerve was preserved in 12 of the 13 patients in whom the nerve was intact preoperatively. With respect to hearing preservation, an analysis of 19 consecutive patients with unilateral acoustic neuromas treated from 1975 to 1981 (included in the 181 cases) showed that hearing preservation was achieved in six $(32 \%)^{4}$. These patients were a consecutive series selected for the suboccipital approach and cochlear nerve preservation according to the criteria outlined in Tables 2 and 3.

With all the approaches, the goal is to be able to visualize the tumour, brainstem (Fig. 1) and surrounding nerves to the greatest extent possible (Figs. 2-4). It is now possible with the combination of microscopy and neurophysiological stimulation and recording techniques to identify the seventh and eighth nerves laterally in the internal auditory canal (Fig. 2) and medially at the brainstem (Fig. 3) in almost every case.

\section{Discussion}

Technological advances have contributed greatly to the current improvement in the results of treating acoustic neuromas. Cushing's book ${ }^{7}$ beautifully outlines the problems faced by the surgeon in that era where large tumours were the rule, and operative difficulties with bleeding, and adherence of the tumours to the brainstem not only frequently prevented complete excision, but produced a considerable mortality rate. With the advent of improved diagnostic methods and operative aids such as coagulation during McKenzie's era', many patients had their tumours completely excised. Drake's ${ }^{2}$ results in the 1960's were even better since the use of the operating microscope allowed tumours to be dissected away from the cranial nerves and brainstem. In the last two decades, numerous technological discoveries have led to further advances in acoustic neuroma surgery. With the

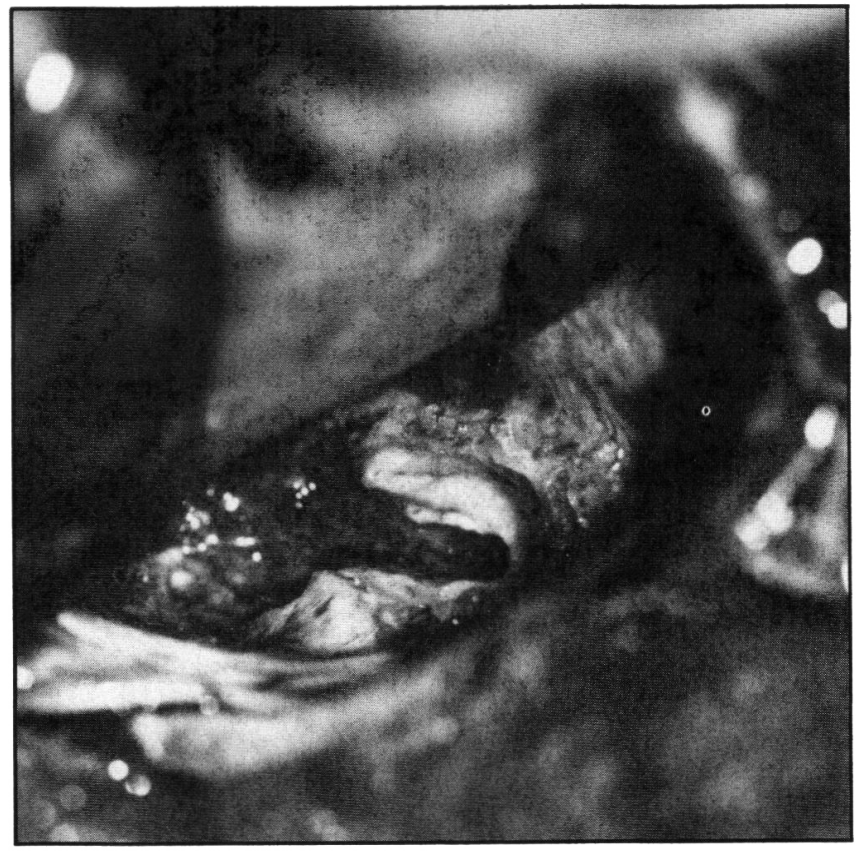

Figure 2 - The bone of the right posterior canal wall has been removed and the dura opened. The nerves are darkened by a thin layer of blood. The retractor on the cerebellum lies above. and the attachment of the tentorium to the petrous ridge can be seen to the right of the retractor. 


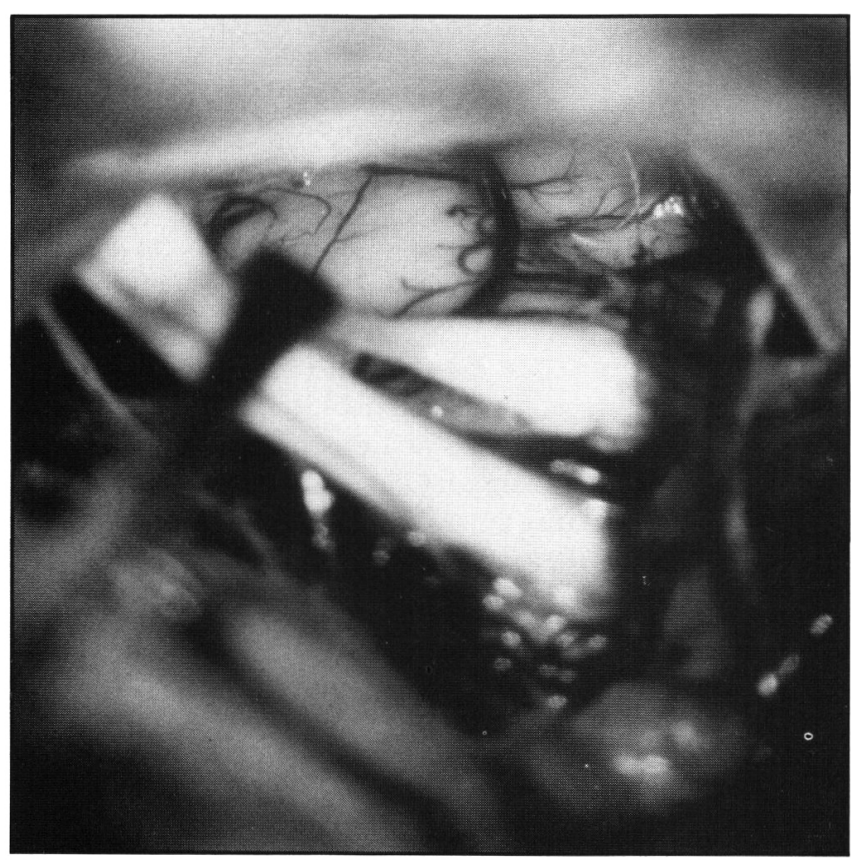

Figure 3 - The right seventh and eighth cranial nerves are seen near the pontomedullary junction. The seventh nerve which is being retracted exits. from the brainstem anteriorly and medially to the eighth nerve. A large vessel is traversing the origin of both nerves (at the right of the photograph).

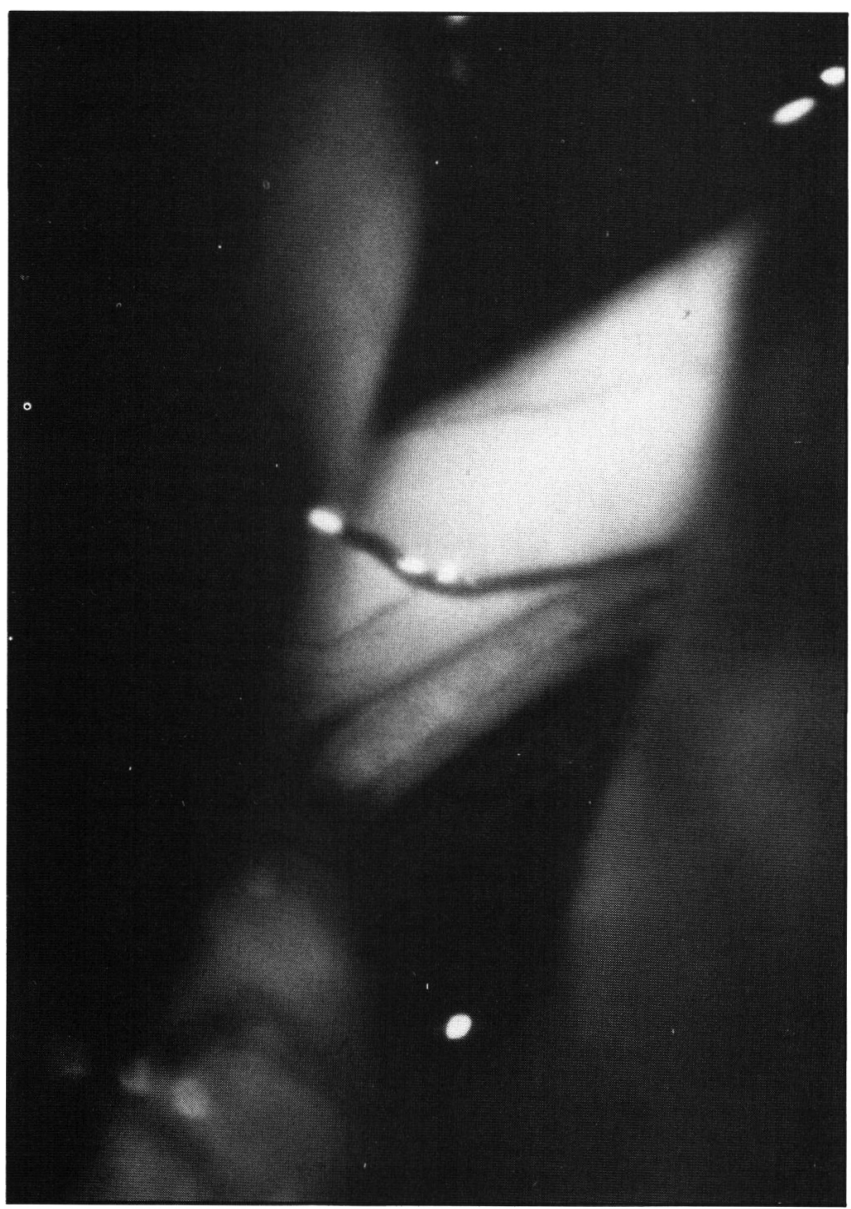

Figure 4 - The right seventh and eighth cranial nerves as they approach the porus acousticus in a patient with an intracanalicular tumour. The seventh nerve is seen slightly anterior to the eighth nerve.
CT scan, tumours in the cerebellopontine angle of $1.0 \mathrm{~cm}$ could be detected and more recently with MR imaging even intracanalicular tumours can be detected ${ }^{8}$. Neurotological investigation has also been improved by the development of brainstem evoked potentials as a diagnostic test ${ }^{9}$. The results of surgery are better now mainly because smaller tumours are being operated upon due to earlier detection, and because of improved intra-operative technology. For example, continuous EMG monitoring of facial muscle action potentials and continuous direct monitoring of cochlear nerve action potentials are of major importance in improving preservation of the seventh and eighth cranial nerves.

A team approach involving a neurosurgeon and neuro-otologist has been developed in several centres and has been an important factor in improving the results. Collaboration increases the number of surgical approaches that can be used, and allows the approach to be indivualized according to the criteria outlined above (Tables 1-3). This approach improves the likelihood of complete excision of small and large tumours with minimal morbidity and mortality, and the likelihood of preservation of the surrounding cranial nerves. Smaller tumours can be excised with almost equal safety and precision from either suboccipital or translabyrinthine approaches. However, only the former allows preservation of the cochlear nerve and hearing. The expanded translabyrinthine approach is better for large tumours especially those growing forward along the midbrain and through the tentorial hiatus. With this approach, these tumours can more often be completely excised, with acceptably low mortality and morbidity, and with a reasonable chance of preserving facial nerve function. Each approach is attended by its own types of complications, a good example of which is CSF rhinorrhea after the translabyrinthine approach. The incidence and treatment of these complications will be the subject of a separate report.

House $^{6}$ reported the translabyrinthine approach in the 1960's for smaller cerebellopontine angle tumours, and initially it was rejected by most neurosurgeons mainly because of its limited visualization of the angle and other areas of the posterior fossa. Since then, neuro-otologists have removed increasing amounts of the petrous temporal bone and have gained a sufficiently large exposure of the angle for removal of large tumours. Morrison and King ${ }^{10,11}$ increased the exposure even further by removing the squamous portion of the temporal bone, thus allowing visualization of the middle fossa. If the tentorium is divided from the superior petrosal sinus to the hiatus, the posterior and middle fossae communicate, allowing visualization of the lateral, anterior and superior aspects of large tumours as previously described ${ }^{3}$. This additional exposure is usually not necessary if the labyrinthectomy is "expanded" to include much more of the petrous temporal bone, superiorly to the middle fossa dura, medially to the anterior lip of the porus, and inferiorly to the sigmoid sinus, jugular bulb, and cochlear aqueduct. Thus, even with large tumours the expanded translabyrinthectomy usually provides sufficient exposure. However, the combined translabyrinthine, middle fossa temporal craniotomy approach with division of the tentorium as previously described ${ }^{3}$ is still occasionally used for exceptionally large tumours with anteromedial or superior extensions, especially in patients with small temporal bones.

The number of meningiomas which mimic acoustic neuromas is of interest. Hopefully, newer diagnostic aids will differ- 
entiate these lesions. The same is true for cases of arachnoiditis with occluded internal auditory canals in whom more accurate preoperative tests would obviate surgical exploration. With meningiomas, there is a better chance of improving cochlear nerve function than with acoustic neuromas. Therefore, with the smaller meningiomas, the suboccipital approach should be used, but with larger tumours the considerations regarding approach are identical to those for the acoustic neuromas.

\section{ACKNOWLEDGEMENTS}

The author is grateful to Mrs. Jean Base for compiling the data upon which this work is based.

\section{REFERENCES}

1. McKenzie KG and Alexander E. Acoustic Neurinoma. Clin Neurosurg 1955; $2: 21-36$

2. Drake CG. Surgical treatment of acoustic neuroma with preservation or reconstitution of the facial nerve. J Neurosurg 1967; 26: 459-464.

3. Tator $\mathrm{CH}$ and Nedzelski JM. Facial nerve preservation in patients with large acoustic neuromas treated by a combined middle fossa transtentorial translabyrinthine approach. J Neurosurg 1982; 57: 1-7.

4. Tator $\mathrm{CH}$ and Nedzelski JM. Preservation of hearing in patients undergoing excision of acoustic neuromas and other cerebellopontine angle tumours. J Neurosurg 1985; 63: 168-174.

5. DiTullio MV, Malkasian D and Rand RW. A critical comparison of neurosurgical and otolaryngological approaches to acoustic neuromas. J Neurosurg 1978; 48: 1-12.

6. House WF ed. Monograph II. Acoustic Neuroma. Arch Otolaryngol 1968; 88: 576-715.

7. Cushing $H$. Tumors of the nervus acusticus and the syndrome of the cerebellopontine angle. Philadelphia and London. W.B. Saunders, 1917.

8. Daniels DL, Herfkius R, Koehler PR, et al. Magnetic resonance imaging of the internal auditory canal. Radiology 1984; 151: 105-108.

9. Moller MB and Moller AR. Brainstem auditory evoked potentials in patients with cereivellopontine angle tumours. Ann Otol Rhinol Laryngol 1983; 92: 645-650.

10. Morrison AW and King TT. Experiences with a translabyrinthine - transtentorial approach to the cerebellopontine angle. J Neurosurg 1973; 38: 383-390.

11. King TT and Morrison AW. Translabyrinthine and transtentorial removal of acoustic nerve tumors. J Neurosurg 1980; 52: 210-216. 